The LAMBDA photon-counting pixel detector and high-Z sensor development

This content has been downloaded from IOPscience. Please scroll down to see the full text.

2014 JINST 9 C12026

(http://iopscience.iop.org/1748-0221/9/12/C12026)

View the table of contents for this issue, or go to the journal homepage for more

Download details:

IP Address: 131.169.233.159

This content was downloaded on 03/02/2015 at 13:16

Please note that terms and conditions apply. 


\title{
The LAMBDA photon-counting pixel detector and high-Z sensor development
}

\author{
D. Pennicard ${ }^{a}{ }^{1}$ S. Smoljanin, ${ }^{a}$ B. Struth, ${ }^{a}$ H. Hirsemann, ${ }^{a}$ A. Fauler, ${ }^{b}$ M. Fiederle,${ }^{b}$ \\ O. Tolbanov, ${ }^{c}$ A. Zarubin, ${ }^{c}$ A. Tyazhev, ${ }^{c}$ G. Shelkov ${ }^{d}$ and H. Graafsma ${ }^{a}$ \\ ${ }^{a} \mathrm{DESY}$, \\ Hamburg, Germany \\ ${ }^{b}$ Albert-Ludwigs-University Freiburg, \\ Freiburg, Germany \\ ${ }^{c}$ Functional Electronics Laboratory of Tomsk State University, \\ Tomsk, Russia \\ ${ }^{d}$ JINR \\ Dubna, Russia
}

E-mail: david.pennicard@desy.de

ABSTRACT: Many X-ray experiments at third-generation synchrotrons benefit from using singlephoton-counting detectors, due to their high signal-to-noise ratio and potential for high-speed measurements. LAMBDA (Large Area Medipix3-Based Detector Array) is a pixel detector system based on the Medipix3 readout chip. It combines the features of Medipix3, such as a small pixel size of $55 \mu \mathrm{m}$ and flexible functionality, with a large tileable module design consisting of 12 chips $(1536 \times 512$ pixels $)$ and a high-speed readout system capable of running at 2000 frames per second.

To enable high-speed experiments with hard X-rays, the LAMBDA system has been combined with different high-Z sensor materials. Room-temperature systems using GaAs and CdTe systems have been produced and tested with X-ray tubes and at synchrotron beamlines. Both detector materials show nonuniformities in their raw image response, but the pixel yield is high and the uniformity can be improved by flat-field correction, particularly in the case of GaAs. High-frame-rate experiments show that useful information can be gained on millisecond timescales in synchrotron experiments with these sensors.

KEYWORDS: Solid state detectors; X-ray detectors; Materials for solid-state detectors; Hybrid detectors

\footnotetext{
${ }^{1}$ Corresponding author.
} 


\section{Contents}

1 The LAMBDA detector system 1

2 LAMBDA with chromium-compensated Gallium Arsenide sensors 2

2.1 Chromium-compensated Gallium Arsenide 2

2.2 Test results 3

2.3 Using the GaAs LAMBDA in synchrotron experiments 4

3 LAMBDA with Cadmium Telluride detectors 5

$\begin{array}{lll}3.1 & \text { Test results } & 6\end{array}$

$\begin{array}{ll}3.2 \text { Detector stability over time } & 6\end{array}$

4 Conclusions $\quad 7$

\section{The LAMBDA detector system}

LAMBDA (Large Area Medipix3 Based Detector Array) is a photon-counting hybrid pixel detector, based on the Medipix 3 chip [1] and designed for use at synchrotrons. Its key features are: effectively zero noise when detecting $\mathrm{X}$-ray photons above $4 \mathrm{keV}$, due to photon counting; a small pixel size of $55 \mu \mathrm{m}$; a large, tileable module layout that can be used to build large-area systems; fast, deadtime-free readout at up to 2000 frames per second; and finally compatibility with high$\mathrm{Z}$ materials. Typically, experiments at synchrotron beamlines study the structure of matter on an atomic scale though techniques such as X-ray diffraction. By combining the extremely high intensity of a synchrotron beamline with the high signal-to-noise, speed and spatial resolution of LAMBDA, it is possible to study rapid changes in structure over time, or to perform a series of experiments more quickly.

The design of the LAMBDA system is described in more detail elsewhere [2, 3]. It is a modular system, with each module consisting of a detector head and power and readout electronics. A single LAMBDA module with a Gallium Arsenide sensor is shown in figure 1. (The GaAs sensor itself is described in the next section.) The detector head consists of a sensor assembly mounted on a ceramic circuit board. The ceramic board is designed to read out either a single large sensor of $85 \mathrm{~mm}$ by $28 \mathrm{~mm}$ bonded to 12 Medipix 3 chips, or one or more smaller sensor assemblies - in the figure, the GaAs sensor is connected to 6 chips. The rest of the readout electronics provide powering to the detector head, control the Medipix 3 chips during operation, and allow communication with a control PC. The readout electronics include four 10 Gigabit Ethernet links, for high-speed readout to the PC. All the boards are designed to fit behind the detector head, so multiple modules can be tiled to cover a larger area.

The Medipix 3 chip itself was developed by CERN on behalf of a collaboration of 20 institutes, and was designed for flexible use in different applications. Each pixel has resources such as a 2stage amplifier, two sets of thresholding circuitry for discriminating photon energy, two 12-bit 


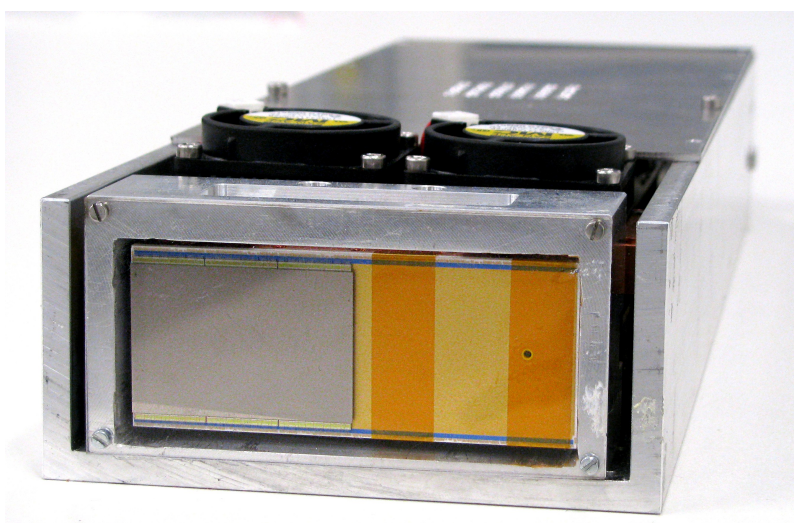

Figure 1. A GaAs(Cr) detector with LAMBDA readout electronics. The sensor has 768 by 512 pixels of 55 $\mu \mathrm{m}$, giving an area of $42 \mathrm{~mm}$ by $28 \mathrm{~mm}$, and a thickness of $500 \mu \mathrm{m}$. The sensor layer is bump bonded to 6 Medipix3 readout chips.

counters and various forms of inter-pixel communication, and these can be configured in different modes. In synchrotron experiments, the chips are primarily used in "continuous read-write" mode. By alternately accumulating photon hits in one counter while reading out the other counter, it is possible to achieve high frame rates with negligible dead time between images. However, it is also possible, for example, to configure the detector to divide photon hits into multiple energy bins, in order to get additional spectroscopic information. This "colour imaging" mode could be used to distinguish between different elements in a sample.

LAMBDA systems with large silicon sensors have been constructed and tested in synchrotron experiments at moderate X-ray energies. However, silicon sensors have low absorption efficiency for photons above $25 \mathrm{keV}$, so silicon modules cannot provide efficient detection in hard X-ray experiments. By replacing the silicon sensor in LAMBDA with a "high-Z" (high atomic number) semiconductor, it would be possible to achieve a combination of extremely high signal-to-noise ratio and high speed even in hard X-ray experiments. This would be particularly valuable at synchrotrons, since it would make it possible to use hard X-rays to study changes in samples on the timescale of milliseconds. However, high- $Z$ sensors also have downsides compared to silicon. Most high- $\mathrm{Z}$ semiconductors are compounds, and cannot be produced with the same level of perfection as silicon. Germanium is an exception, but it needs to be cooled during operation, and is more vulnerable to damage than silicon during processing and operation. To meet the goal of providing high-Z sensors to synchrotron beamlines, we are working to build and test LAMBDA systems with different materials: Gallium Arsenide, Cadmium Telluride and Germanium. This paper focuses on the performance of larger-area GaAs and CdTe sensors.

\section{LAMBDA with chromium-compensated Gallium Arsenide sensors}

\subsection{Chromium-compensated Gallium Arsenide}

Gallium arsenide is a widely-used semiconductor material in applications such as opto-electronics. The higher atomic number of this material makes it appealing for hard X-ray detection, and its 
high electron mobility potentially makes it a faster material than silicon. However, as with many compound semiconductors, it is difficult to produce thick GaAs wafers with the combination of properties required for use in a high-performance pixel detector: high resistivity (so that when biased, there is a strong electric field throughout the sensor volume and low leakage current); long enough carrier lifetimes to ensure high charge collection efficiency; and high uniformity. GaAs ingots grown in an arsenic-rich environment achieve high resistivity, but contain defects that lead to high electron trapping. Conversely, in n-type wafers the excess electrons fill these defects and improve the electron lifetime, but this naturally leads to low resistivity.

One approach to producing detector-grade GaAs is to alter the properties of the material by doping. An effective way of doing this has been developed by Ayzenshtat et. al. in 2001, where standard n-type GaAs wafers are doped with $\mathrm{Cr}$ by diffusion [4]. The $\mathrm{Cr}$ compensates the excess electrons in this material, thus achieving high enough resistivity to allow it to be used as a photoconductor detector with ohmic contacts.

A LAMBDA module with a $\mathrm{GaAs}(\mathrm{Cr})$ sensor was produced as part of a German-Russian collaboration project, GALAPAD, between FEL (Functional Electronics Laboratory of Tomsk State University), JINR (Dubna), the University of Freiburg, the Karlsruhe Institute of Technology, and DESY. The sensor was produced by FEL, and consisted of a $500 \mu \mathrm{m}$-thick layer of $\mathrm{GaAs}(\mathrm{Cr})$ with $55 \mu \mathrm{m}$ pixels. The sensor had an array of 768 by 512 pixels, with a sensitive area of $42 \mathrm{~mm}$ by $28 \mathrm{~mm}$. This layout corresponds to 3 by 2 Medipix 3 chips. The sensor was bonded to the readout chips by the University of Freiburg using PbSn bump bonds [5]. The assembly was then mounted on a LAMBDA circuit board with silicone glue, wire bonded and attached to readout electronics. For cooling, the circuit board was mounted on a copper block, which in turn was cooled by a pair of fans. The system was then placed inside a housing for convenient operation. The finished detector module is shown in figure 1 .

\subsection{Test results}

For testing, the GaAs sensor was connected to a bias voltage of $-300 \mathrm{~V}$ and operated with the chip in electron-readout mode. Because the pixel size is small compared to the sensor thickness, most of the measured signal in each pixel will be due to the carriers drifting towards the pixelated side, rather than the back contact. This so-called "small pixel effect" is discussed in [6]. In Crcompensated GaAs, it has been shown that electrons have a higher lifetime-mobility product than holes, largely due to the inherently higher mobility of electrons in GaAs, and this means that electron readout will give higher collection efficiency [7]. During operation, the sensor had a leakage current of $200 \mu \mathrm{A}$ at a temperature of approximately $35^{\circ} \mathrm{C}$.

In order to test the performance of the sensor material, it was first necessary to perform an equalisation procedure to compensate for pixel-to-pixel variations in the Medipix 3 readout chips, and then to perform energy calibration of each chip so that all the chips can be reliably set to the same threshold value. These steps are required when working with Medipix3 regardless of the choice of sensor material, and are discussed, for example, in ref. [8].

Once the calibration was performed, the detector was uniformly illuminated with X-rays from a Molybdenum target X-ray tube at $50 \mathrm{kV}$, and a series of 30 images were taken, each for 30 . The images were summed and normalised to the average pixel value, and the resulting image is shown in figure 2A. The raw image shows a high level of nonuniformity, with a granular structure which 

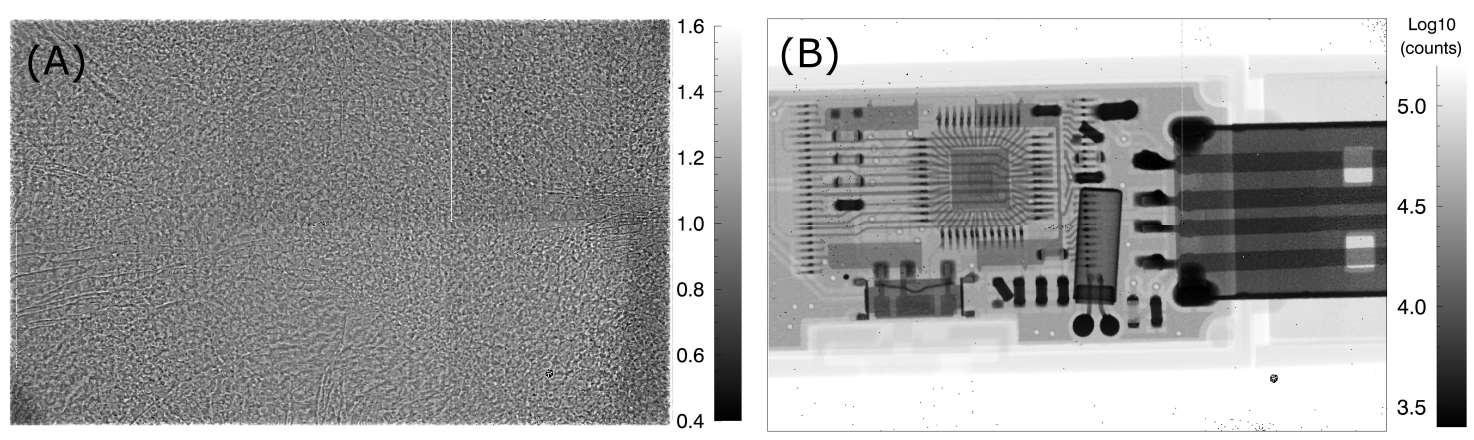

Figure 2. (A) Image of the response of the GaAs LAMBDA module to uniform illumination with X-rays from a Mo tube at 50kV, using $-300 \mathrm{~V}$ bias voltage and $10 \mathrm{keV}$ threshold. (B) Flat-field corrected image of a USB stick taken with the GaAs LAMDBA module. The image is logarithmically scaled to show the large contrast range clearly.

is probably related to the original growth process of the wafer. The standard deviation of the pixelto-pixel variation (excluding dark and noisy pixels) is $21.0 \%$.

However, by applying flat-field correction (i.e. using the flat-field response to correct subsequent images), the image quality can be greatly improved. Figure $2 \mathrm{~B}$ shows a flat-field corrected $\mathrm{X}$-ray image of a USB stick. The imaging conditions were the same as for the flat-field image, and the image is shown with a logarithmic colour scaling. The image shows a good quality, with a high yield of functional pixels. Excluding a row of pixels that are nonfunctional due to a problem with the readout chip rather than the sensor, there are 640 dark pixels $(0.16 \%)$ and 300 noisy pixels $(0.08 \%)$, giving $99.76 \%$ working pixels. To test the effectiveness of the flat-field correction more quantitatively, a second set of flat-field images were taken as above, and the first set of flat-field data was used to correct them. This reduced the standard deviation of the pixel-to-pixel variation (excluding dark and noisy pixels) from $21.0 \%$ to $0.15 \%$. It has also been shown elsewhere that the detector response is stable over time, which means that after the flat-field measurement is acquired it can be consistently used to correct the detector response [9]. It should be noted that flat-field correction factors are dependent on photon energy, and when an object is imaged with polychromatic $\mathrm{X}$-rays the transmitted spectrum will differ from the direct tube spectrum, thus reducing the accuracy of flat-field correction. This, however, is not a problem for experiments with monochromatic beam at synchrotrons.

\subsection{Using the GaAs LAMBDA in synchrotron experiments}

A high proportion of experiments at synchrotrons use X-ray scattering to study a sample's structure on an atomic scale. Using hard X-rays for these experiments makes it possible to gain higher spatial resolution, to study large samples or samples made of heavier elements, and to probe samples while they are contained in sample enviroments. A good example of the latter would be extreme conditions experiments. By compressing a sample inside a diamond anvil cell while heating it with a laser, it is possible to recreate the extremely high pressures and temperatures found inside of planets or in the outer layers of stars. By probing the sample with the X-ray beam, it is possible to learn more about how matter behaves under these conditions. High-speed photon counting 

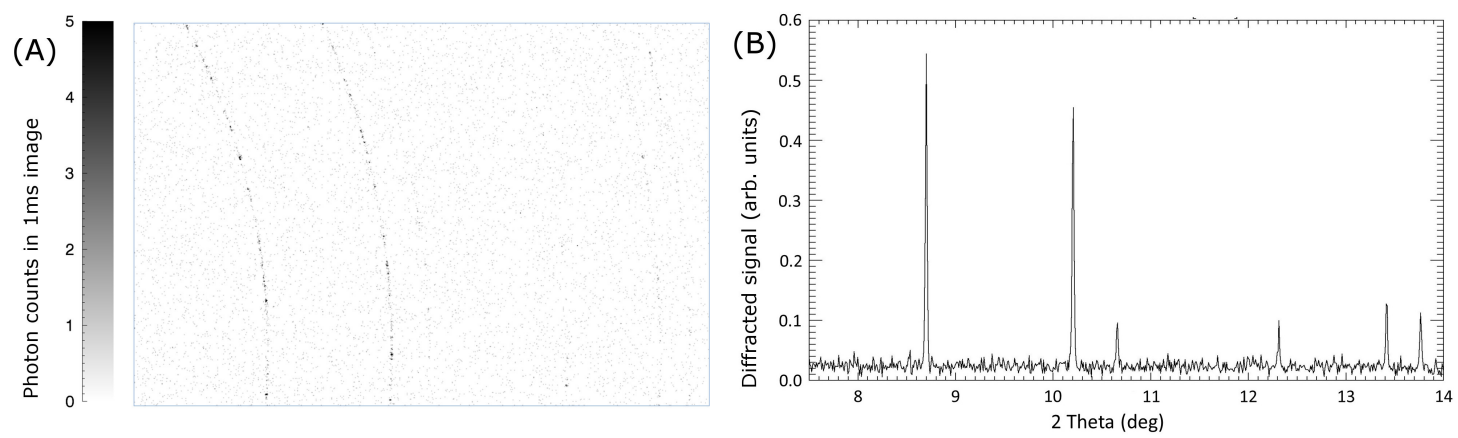

Figure 3. (A) Diffraction pattern obtained in $1 \mathrm{~ms}$ with the GaAs LAMBDA detector. The sample was $\mathrm{CeO}_{2}$ powder in a diamond anvil cell, illuminated by a $42 \mathrm{keV}$ synchrotron X-ray beam at PETRA-III P02.2. (B) The diffracted X-ray intensity integrated as a function of scattering angle, using the single 1ms image shown in $\mathrm{A}$.

detectors like LAMDBA should then allow scientists to study the rapid changes that occur in a sample when the pressure and temperature are changed.

To investigate this possibility, the GaAs LAMBDA detector was tested at PETRA-III beamline P02.2, which is used for extreme conditions experiments. A sample of a common test standard, $\mathrm{CeO}_{2}$, was placed inside a diamond anvil cell in the X-ray beam. The X-ray beam had a photon energy of $42 \mathrm{keV}$; at this energy, the photoelectric absorption efficiency of $500 \mu \mathrm{m}$-thick GaAs will be $74 \%$, compared to $4.6 \%$ for the same thickness of silicon. The LAMBDA GaAs detector was placed at a distance of $35 \mathrm{~cm}$, with a horizontal offset of $4 \mathrm{~cm}$ between the X-ray beam and the edge of the sensitive area. A series of images were then taken with different shutter times. Figure 3A shows an image taken with a shutter time of $1 \mathrm{~ms}$. Because the sample is a coarse powder, the diffraction pattern consists of rings of diffraction spots, with each spot produced by scattering from a single grain of the material. The angle and intensity of these rings convey information about the crystalline structure of the material. Due to the short shutter time, the rings are faint, with most pixels only detecting a few photons. Nevertheless, due to the effectively noise-free behaviour of the detector, when the photon hits in the $1 \mathrm{~ms}$ image are integrated as a function of scattering angle a clear diffraction signal is obtained, with the rings clearly distinguished from the background level, as shown in figure 3B. This demonstrates that the detector could be used to measure structural changes in a sample on a timescale of milliseconds.

\section{LAMBDA with Cadmium Telluride detectors}

Cadmium Telluride is a well-established detector material, which is now commercially available in 3" wafers. These wafers provide both good resistivity and carrier lifetime, though they still have some nonuniformities. Since it is a more brittle material than GaAs, the risk of damaging the material during bump bonding is greater. The biggest benefit of CdTe is that it has substantially higher quantum efficiency at extreme energies, which makes it a natural choice for experiments at $80 \mathrm{keV}$ and above. However, CdTe produces fluorescence photons around $25-30 \mathrm{keV}$, so if the incoming X-rays are in the range of about $25-50 \mathrm{keV}$ these fluorescence photons can both reduce 
the signal by escaping from the detector and blur the image by being re-absorbed in neighbouring pixels. So, there are advantages of being able to choose different high- $Z$ materials for different energy ranges.

A LAMBDA module was built with a CdTe sensor. The sensor itself was made from $1000 \mu \mathrm{m}-$ thick material from the company Acrorad. At the University of Freiburg, ohmic pixel contacts were produced on the CdTe wafer with a $55 \mu \mathrm{m}$ pitch. The sensor layout corresponded to 6 Medipix 3 chips, giving a pixel array of 768 by 512 and an area of $42 \mathrm{~mm}$ by $28 \mathrm{~mm}$, the same as the GaAs module. The sensor was bump-bonded to the Medipix 3 chips with PbSn bumps. The finished sensor was integrated into a LAMBDA module, as described above for the GaAs module.

\subsection{Test results}

The CdTe detector was tested with $-300 \mathrm{~V}$ bias and electron readout. When the bias was first applied, the leakage current was initially high and then decreased over a period of roughly 10 minutes to settle at a value of $65 \mu \mathrm{A}$. The temperature of the sensor during operation was approximately $35^{\circ} \mathrm{C}$. As with the GaAs module, the detector was uniformly illuminated with a Mo target X-ray tube at $50 \mathrm{kV}$, while using a threshold setting of $10 \mathrm{keV}$. The resulting image is shown in figure 4A. The flat field image shows a pattern of lines superimposed over a more uniform response. These lines correspond to dislocations in the sensor material, and show a high-counting region in the centre of the line, and lower counts towards the edges. Additionally, in some places there are "blobs" of insensitive pixels, typically surrounded by rings of pixels with increased counts. These clusters of insensitive pixels are mostly flooded with leakage current, rather than disconnected. Excluding dark and noisy pixels, the standard deviation of the pixel-to-pixel variation is $7.5 \%$, which is better than the GaAs detector.

A USB stick was then imaged with the X-ray tube, and flat-field correction applied to the image. The result is shown in figure 4B. Once again, flat-field correction significantly improves the uniformity of the image, though the "blobs" of insensitive pixels cannot be corrected this way. There are 1116 dark pixels $(0.28 \%)$ and 60 noisy pixels $(0.015 \%)$, giving $99.70 \%$ functional pixels. This is $0.06 \%$ lower yield than the GaAs module, and since the insensitive pixels are more clustered they are also more visible in the image. Once again, a second data set was taken with flat illumination, and then corrected with the first set of flat images. The standard deviation of the pixel-to-pixel variation after correction (excluding dark and noisy pixels) was $0.6 \%$, which is higher than for GaAs after correction. In the corrected images, the network of lines was still faintly visible, indicating that the detector response had changed somewhat from the first to the second set of flat images. This change could be due to both the 10-minute time gap between the measurement sets, and also the irradiation of the sensor. This is a known problem with $\mathrm{CdTe}$, and is investigated in the next section.

\subsection{Detector stability over time}

One downside of CdTe is that its behaviour can change over time, due to polarisation of the material. This CdTe module used ohmic pixel contacts, which typically suffer less from polarization than Schottky devices, but these effects can still occur over extended time periods or after irradiation [10]. To investigate this, during a beamtime at ESRF beamline ID15C the CdTe module was 

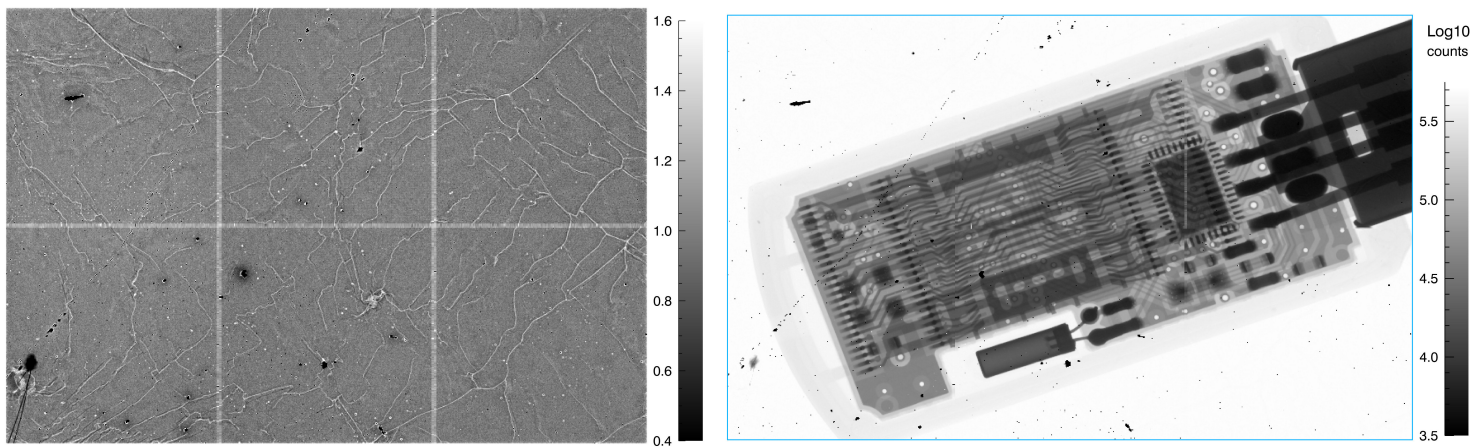

Figure 4. (A) Image of the response of the CdTe LAMBDA module to uniform illumination with X-rays from a Mo tube at 50kV, using $-300 \mathrm{~V}$ bias voltage and $10 \mathrm{keV}$ threshold. (B) Flat-field corrected image of a USB stick taken with the CdTe LAMDBA module. The image is logarithmically scaled to show the large contrast range clearly.

set up inside the hutch in a position where it was exposed to scattered X-ray photons at $70 \mathrm{keV}$. The photon hit rate was low — the detector measured about 100 counts / pixel / second — and the irradiation was uniform across the detector. Images were taken continuously over a period of 10 hours using a shutter time of $30 \mathrm{~s}$, with the detector set to $30 \mathrm{keV}$ threshold and $-300 \mathrm{~V}$ bias.

Firstly, it was found that the overall count rate on the detector increased by $4 \%$ over the first hour of operation, before levelling out and becoming uniform over time. As noted previously, when the high voltage is applied to the sensor the current is initially high, then settles down over time, so this is another indication that the behaviour of the material takes time to reach a state of equilibrium.

Additionally, the changes in flat-field response over time were found. Figure 5 shows the change in flat-field image in a region of the sensor over the first hour of operation, then the following nine hours. This change was calculated by taking the later flat-field response (averaged over a 5 minute period for good statistics) and subtracting the earlier response. Over the period from the start of the measurement to 1 hour, not only is there an overall increase in counts, but also an intensification of the dislocation lines, with the high-count region in the centre increasing further and the low-count region at the edge decreasing. Then, over the period from 1 hour to 10 hours, the overall count rate remains the same, but the intensity of the defect pattern is reduced somewhat. These results imply that a single flat-field measurement is not sufficient to fully correct for the nonuniformites in the detector over an extended period of time. The fact that the change is most rapid after initially switching on the high voltage also implies that rather than frequently resetting the bias, it is helpful to allow the detector to stabilize before making measurements.

\section{Conclusions}

Gallium Arsenide and Cadmium Telluride pixel detectors with $42 \mathrm{~mm}$ by $28 \mathrm{~mm}$ area and 55 $\mu \mathrm{m}$ pixel size have been bonded to Medipix 3 chips and read out by LAMBDA readout electronics. While these materials show non-uniformities in response, this can be improved by flat-field 
(A) - Change from $0 \mathrm{~h}$ to $1 \mathrm{~h}$

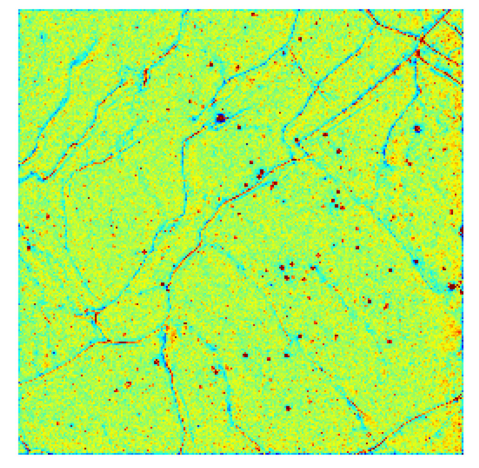

(B) - Change from $1 \mathrm{~h}$ to $10 \mathrm{~h}$
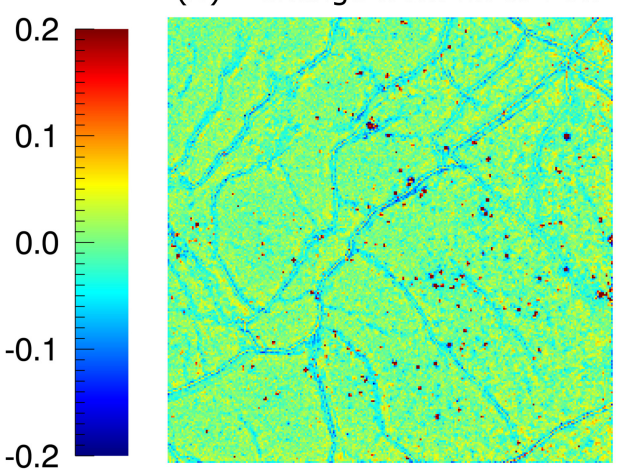

Figure 5. (A) Change in the flat-field response of the CdTe detector to $70 \mathrm{keV}$ photons over the first hour of operation. The plot shows the flat-field response after 1 hour minus the initial flat-field response, scaled so that 1 corresponds to the average response after 1 hour. (B) Change in the flat-field response over the following 9 hours, i.e. from 1 hour into the measurement to 10 hours.

correction, particularly in the case of GaAs. Synchrotron tests have shown that these detectors have sufficient speed and signal-to-noise ratio to produce useful $\mathrm{X}$-ray diffraction images on a millisecond timescale. The next step in this development will be to tile together multiple high- $\mathrm{Z}$ sensors to provide a larger-area detector - for example, 6 of these sensors could be built into a 3-module LAMBDA system with over 2 megapixels. This could then be used to measure a larger image or diffraction pattern on short timescales, in order to perform high-speed or timeresolved experiments.

\section{Acknowledgments}

The authors would like to thank: the Bundesministerium fur Bildung und Forschung (BMBF) for funding the GALAPAD project; Andre Rothkirch, Yuelong Yu, Igor Sheviakov, Qingqing Xia, Milija Sarajlic and Jiaguo Zhang at DESY for general work on LAMBDA; PETRA-III P02 beamline staff Zuzana Konopkova, Emma McBride and Hanns-Peter Liermann; and the ESRF detector group and ID15 staff.

\section{References}

[1] R. Ballabriga et al., The Medipix3RX: A high resolution, zero dead-time pixel detector readout chip allowing spectroscopic imaging, 2013 JINST 8 C02016.

[2] D. Pennicard et al., The LAMBDA photon-counting pixel detector, J. Phys. Conf. Ser. 425 (2013) 062010 .

[3] D. Pennicard et al, Development of LAMBDA: Large Area Medipix-Based Detector Array, 2011 JINST 6 C11009.

[4] G.I. Ayzenshtat et al., GaAs structures for X-ray imaging detectors, Nucl. Instrum. Meth. A 466 (2001) 25. 
[5] M. Fiederle, H. Braml, A. Fauler, J. Giersch, J. Ludwig, G. Anton and K. Jakobs, Development of flip-chip bonding technology for (Cd,Zn)Te, IEEE Nucl. Sci. Symp. Conf. Rec. 1 (2003) 232.

[6] P. Sellin, Modelling of the small pixel effect in gallium arsenide X-ray imaging detectors, Nucl. Instrum. Meth. A 434 (1999) 75.

[7] G.I. Ayzenshtat et al., GaAs resistor structures for X-ray imaging detectors, Nucl. Instrum. Meth. A 487 (2002) 96.

[8] M. Walsh et al., Improving and characterising the threshold equalisation process for multi-chip Medipix 3 cameras in single pixel mode, IEEE Nucl. Sci. Symp. Med. Imag. Conf. (2011) 1718.

[9] E. Hamann, Characterization of High Resistivity GaAs as Sensor Material for Photon Counting Semiconductor Pixel Detectors, PhD thesis, University of Freiburg (2013) www.freidok.uni-freiburg.de/volltexte/9355/pdf/Dissertation_EHamann_korr.pdf.

[10] M. Ruat and C. Ponchut, Defect signature, instabilities and polarization in CdTe X-ray sensors with quasi-ohmic contacts, 2014 JINST 9 C04030. 